DOI: http://dx.doi.org/10.15517/rce.v1i1.29096

\title{
BALANCE FISCAL ESTRUCTURAL COSTA RICA 1998 - 2014
}

\begin{abstract}
RESUMEN
En este artículo se desarrolla un balance fiscal estructural para la economía costarricense. Se propone utilizar un indicador alternativo ex-post de la política fiscal que contribuya a la formulación de una regla fiscal. Para este fin, se utilizó la metodología general del FMI con datos obtenidos del Ministerio de Hacienda de Costa Rica. Además, se desarrolló un modelo de MCO con el propósito de calcular las elasticidades de los ingresos tributarios y del gasto corriente con respecto a la producción nacional. El filtro Hodrick - Prescott fue utilizado para calcular el producto potencial. Se comprobó empíricamente que, para el caso costarricense, el balance fiscal estructural se comporta de la misma manera que la brecha entre el producto efectivo y el potencial.
\end{abstract}

PALABRAS CLAVE: FINANZAS, ESTADO, INGRESO, GASTOS, PRESUPUESTO, CRECIMIENTO ECONÓMICO, POLITICAL FISCAL, ESTADO FINANCIERO.

\begin{abstract}
This article presents a structural fiscal balance for the Costa Rican economy. The structural balance is an ex-post indicator of fiscal policy performance which attempts to contribute to the development of a fiscal rule. In order to derive this indicator the IMF's general methodology was applied to data from the Costa Rican Ministry of Finance. The elasticities of tax revenues and current expenditure to national output were estimated by means of a simple OLS model while potential output was estimated by means of the Hodrick-Prescott. The article's main conclusion is that the structural fiscal balance moves in the same direction as that of the output gap.
\end{abstract}

KEYWORDS: FINANCE, STATE, INCOME, EXPENDITURE, BUDGET, ECONOMIC GROWTH, FISCAL POLICY, FINANCIAL STATEMENT.

1 Universidad Latina de Costa Rica, Escuela de Economía; Código Postal 1561-2050; San José, Costa Rica; rojas22daniel@gmail.com Moody's Analytics; Código Postal 3006; Heredia, Costa Rica. 


\section{INTRODUCCIÓN}

Los resultados fiscales convencionales, es decir, aquellos que incluyen el componente cíclico en su resultado, son indicadores limitados de la política fiscal costarricense, dada su relación con el ciclo económico, ya que, como se comprueba en esta investigación, los ingresos y gastos del Gobierno Central costarricense no dependen exclusivamente de las decisiones tomadas bajo medidas discrecionales. Los ingresos tributarios y gastos corrientes dependen, en parte, de las fluctuaciones del ciclo económico, lo que puede distorsionar nuestra percepción del balance fiscal y en consecuencia, las posibles acciones de intervención por parte del gobierno (ver gráfico 1).

Debido a lo anterior, se propone construir un indicador de balance estructural que elimine, del balance convencional, el componente cíclico ajeno a la autoridad económica. Este indicador es una medición ex-post del resultado fiscal, la cual refleja el nivel de ingresos y gastos si la economía se hubiese comportado conforme a la tendencia de largo plazo. De igual manera, este indicador puede ser de utilidad al gobierno para distinguir si es necesario un cambio en la política fiscal -en términos de su orientación expansiva o contractiva- para lograr los objetivos de mediano plazo, así como su orientación mediante el uso discrecional de ciertos ingresos y gastos.

\section{GRÁFICO 1}

COSTA RICA: VARIACIÓN INTERANUAL DE INGRESOS

TRIBUTARIOS, GASTO CORRIENTE Y PIB REAL DE 1998 AL 2014

(EN PORCENTAJES)

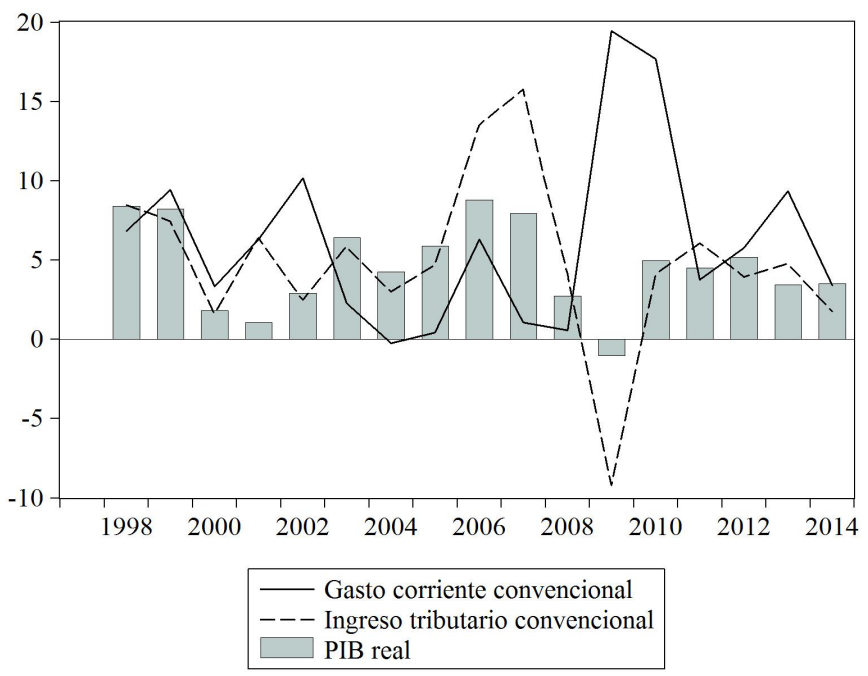

Fuente: Elaboración propia con base en información del Ministerio de Hacienda y el Banco Central de Costa Rica.

La metodología aplicada en esta investigación es utilizada por el Fondo Monetario Internacional (FMI) para obtener el indicador de balance estructural en países desarrollados. Este cálculo siempre viene incluido en las ediciones del World Economic Outlook (WEO)1. El trabajo de Hagemann (1999) es la base de la metodología utilizada por el FMI. La Organización para la Cooperación y el Desarrollo Económico utiliza una metodología similar para establecer el balance estructural de sus países miembros. 
En el presente estudio se aplica por primera vez la metodología del FMI para estimar un indicador de balance estructural para el Gobierno Central de Costa Rica. Dada la elaboración de este indicador, el autor desea resaltar tres diferentes hallazgos:

- Primero: los resultados indican que, para Costa Rica, el resultado fiscal estructural se comporta de la misma forma que la brecha observada entre el producto efectivo y su potencial. Es decir, a medida que crece (en valor absoluto) una brecha del producto negativa, el balance estructural reducirá su superávit o bien, se ampliará el déficit.

- Segundo: así como las metodologías varían entre las investigaciones, los resultados también lo hacen. Cuando se contrastó la metodología empleada en esta investigación con la de Armendáriz (2006), se encontraron diferencias metodológicas que pueden originar diferencias en los resultados para el periodo en que ambas investigaciones coinciden (1998 al 2003). En este estudio se obtiene un déficit primario estructural, mientras que Armendáriz (2006) muestra un superávit primario estructural. La investigación de Ardanaz, Corbacho, Gonzáles y Caballero (2015) para el periodo 1998 al 2005, también obtiene un superávit primario estructural. El resultado de un superávit estructural para los periodos mencionados se considera desacertado debido a dos razones:

a. En el presente estudio se muestra que el balance estructural y el balance convencional presentaron un comportamiento muy similar, lo que implica un improbable escenario superavitario, debido a la existencia de un fuerte componente estructural.

b. Para el periodo 2001 - 2005, esta investigación determinó la existencia de una brecha del producto negativa, la cual -de acuerdo con el punto anterior- llevaría a un déficit en el resultado fiscal estructural o a una reducción del superávit estructural, diferente a lo observado en los hallazgos de Armendáriz (2006) y Ardanaz et al. (2015).

Debe mencionarse además que el estudio de Martner (2000) se centró en el sector público no financiero costarricense, por lo que sus resultados no son comparables con los de la presente investigación.

- Tercero: los resultados obtenidos en esta investigación tienen una relación estrecha con las cifras fiscales utilizadas para el cálculo del indicador. Se utilizaron cifras a cierre de año, publicadas en la página oficial del Ministerio de Hacienda de Costa Rica. Una estimación del balance estructural para Costa Rica que utilice cifras del presupuesto nacional, llevaría a resultados inexactos. Esto se debe a que el presupuesto nacional costarricense sufre modificaciones (subejecuciones y presupuestos extraordinarios), realizadas por la Asamblea Legislativa a lo largo del año, lo que resulta en un presupuesto ejecutado diferente del que se aprobó inicialmente. Se podría pensar en utilizar el último presupuesto aprobado por la Asamblea Legislativa sin embargo, esto deja de lado las partidas sobre-ejecutadas o subejecutadas por parte del Poder Ejecutivo. Para el caso de Hagemann (1999), este llama al indicador "Balance Presupuestario Estructural"2, debido a que para sus estimaciones, utiliza las cifras de los presupuestos nacionales de los países desarrollados del FMI.

1 En el momento en que se realizó este trabajo investigativo, la última publicación de la base de datos del WEO (International Monetary Fund, 2015) muestra que el indicador de balance estructural no se encuentra calculado para Costa Rica.

2 La razón por la cual el presente documento no lleva como nombre "Balance Presupuestario Estructural" como en Hagemann (1999), deriva de evitar confusiones a la hora de relacionar el título del presente documento con las cifras fiscales utilizadas para el cálculo del indicador. 
Finalmente, como menciona Marcel et al. (2001), el balance estructural es un indicador simple y entendible para el público en general, profundiza el análisis del desempeño fiscal del Gobierno central y es una herramienta fundamental para la elaboración de reglas fiscales. Tales reglas buscan asegurar la sostenibilidad a largo plazo en el manejo de las finanzas públicas y permitir a las autoridades económicas enfrentar futuros compromisos fiscales. Asímismo, es importante mencionar que, dada la relación de estas reglas fiscales con el ciclo económico, su aplicación puede comprometerse ante periodos de recesión o sobrecalentamiento de la economía. Es por esta razón que el gobierno debe, a la hora de formular dichas reglas fiscales, establecer las excepciones para tales casos, donde se defina cuando y en qué magnitud podría violarse la regla.

La presente investigación se estructura de la siguiente manera; en la siguiente sección se presentan algunos antecedentes, las secciones III y IV presentan la metodología utilizada y los resultados, respectivamente, y posteriormente en la sección V se elaboran las consideraciones finales de la investigación.

\section{ANTECEDENTES}

Las contribuciones iniciales en la elaboración de un indicador de balance estructural para Costa Rica vienen de Méndez y Durán (1995) y Azofeifa y Rojas (2000). El primero determinó cuánto del balance fiscal obedece al entorno macroeconómico y cuánto a las medidas discrecionales de la autoridad fiscal. El cálculo se enfocó en el sector público combinado (Gobierno Central, Banco Central e instituciones no financieras), abarcando el periodo de 1987 a 1994. Méndez y Durán (1995) realizaron sus estimaciones con la metodología de impulso fiscal ${ }^{3}$, elaborada por el FMI. En sus resultados, se determinó la existencia de un balance estructural deficitario y un componente cíclico electoral. El segundo documento utilizó una metodología elaborada por la Comisión Económica para América Latina y el Caribe (CEPAL) para determinar el balance cíclico y estructural en el periodo de 1983 a 1999. Azofeifa y Rojas (2000), concluyen que para el sector público no financiero y para el Gobierno Central, el resultado fiscal se vio influenciado por un fuerte componente estructural, específicamente, determinaron que la deuda interna es el componente estructural que más influyó en el balance primario estructural.

Otras investigaciones que estimaron un indicador de balance estructural para la economía costarricense fueron; Martner (2000), Armendáriz (2006) y Ardanaz et al. (2015) . La primera estimó un balance estructural para el sector público no financiero costarricense para el periodo de 1990 a 1999. Mientras que Armendáriz (2006) y Ardanaz et al. (2015) centraron su análisis en el Gobierno Central, comprendiendo los periodos de 1990 a 2003 y de 1997 a 2013, respectivamente. Martner (2000) y Armendáriz (2006) aplicaron la metodología elaborada por la Comisión Europea, la cual utiliza un componente $\alpha$, que representa una sensibilidad del saldo público a la brecha del producto y un parámetro $\beta$, que representa el componente fijo estructural del saldo público. Por otro lado, Ardanaz et al. (2015) emplearon la metodología de Bornhorst, Dobrescu, Fedelino, Gottschalk and Nakata (2011). En esta metodología se multiplica el componente del gasto por un factor $\left(\frac{Y^{P}}{Y}\right)^{\varepsilon_{G}}$, donde $Y^{P}$ es el Producto Interno Bruto (PIB) potencial, $Y$ es el PIB efectivo $y, \varepsilon_{\mathrm{G}}$ es la elasticidad del gasto con respecto a la producción nacional. Siguiendo a Basso (2006), la utilización de un factor como $\left(\frac{Y^{P}}{Y}\right)^{\varepsilon}$ aplica únicamente para aquellos componentes que fluctúan conforme al ciclo económico.

3 Para una descripción más detallada de esta metodología, ver Schinasi \& Lutz (1991). 


\section{METODOLOGÍA}

A continuación, se presentan los datos utilizados y su respectivo tratamiento.

\section{Datos}

Todos los datos utilizados son anuales y abarcan el periodo de 1998 a 2014. Las cifras fiscales -en millones de colones corrientes-, se tomaron de la base de datos de Ministerio de Hacienda de Costa Rica (s.f). Las cifras utilizadas se determinan "sobre la línea" es decir, no se contemplan fuentes de financiamiento.

Estas cifras fiscales presentan la siguiente estructura:

1. Ingresos totales

a) Ingresos corrientes

- Ingresos tributarios

- Ingresos no tributarios

- Transferencias corrientes

2. Gasto total

a) Gasto corriente

b) Gasto de capital

El PIB real, en millones de colones de 1991, se tomó de la base de datos de Banco Central de Costa Rica (s.f).

La metodología aplicada requiere la utilización de cifras en términos reales, dado que las razones del balance estructural son con respecto al PIB potencial. Por esta razón, en este estudio se deflactaron las cifras fiscales publicadas de forma nominal por el Ministerio de Hacienda de Costa Rica.

En esta investigación se deflactaron las cifras fiscales utilizando el Índice de Precios al Consumidor (IPC) base junio 2006. El mismo se reindexó al año 1991 dado que el PIB real de la economía costarricense es base 1991. Se utilizó el IPC para evitar sobre-estimaciones producto de factores exógenos, logrando así reflejar así el verdadero costo de vida. Por ejemplo, una caída en el precio internacional de los combustibles.

\section{Modelo del Balance Estructural}

Siguiendo a Hagemann (1999), se procede a cuantificar el componente cíclico y discrecional de los ingresos y gastos del Gobierno Central, con el fin de calcular el balance estructural. A continuación se utiliza $B_{t}$ para denotar el balance del Gobierno Central en el año $t$, el cual contiene un factor transitorio y uno permanente, como se expresa a continuación:

$$
B_{t}=B_{C, t}+B_{S, t}=R_{t}-E_{t}
$$


Los subíndices $C$ y $S$ representan el factor cíclico y estructural, respectivamente; el balance $B_{t}$ , por otra parte es la brecha entre ingresos, $R_{t}$, y gastos, $E_{t}$, entonces es posible replantear (1) como:

con

$$
B_{t}=\left(R_{C, t}+R_{S, t}\right)-\left(E_{C, t}+E_{S, t}\right)
$$

$\mathrm{y}$,

$$
R_{t}=R_{C, t}+R_{S, t}
$$

$$
E_{t}=E_{C, t}+E_{S, t}
$$

Según Basso (2006), para determinar el componente estructural de los ingresos fiscales, se parte del supuesto de que existen recursos dentro de los ingresos del Gobierno que se ven afectados por el nivel de actividad $y$ otros que son independientes del mismo; estos últimos no se ven afectados por el ciclo. Con el fin de estimar la posible recaudación tributaria al nivel del producto potencial, es necesario estimar la forma en que el componente cíclico de dicha recaudación varía cuando se modifica la producción nacional.

Siguiendo a Basso (2006), la siguiente regresión se puede utilizar para estimar la elasticidad de los ingresos tributarios con respecto a la producción nacional:

$$
\operatorname{Ln}\left(R_{i, t}\right)=\alpha+\varepsilon_{i} \operatorname{Ln}\left(Y_{t}\right)+\delta_{i, t}
$$

donde $Y_{t}$ es el producto real observado, $R_{i, t}$ denota los ingresos tributarios en términos reales ${ }^{4}, \varepsilon_{i}$ representa la elasticidad de los ingresos tributarios con respecto a la producción nacional y $\delta_{i, t}$, denota una variable dummy, que captura el efecto de la crisis financiera y toma el valor de 1 en los años 2008 y 2009 y 0 en todos los restantes años. En el Anexo 1 se puede apreciar el resultado de la estimación.

Por tanto, siguiendo a Basso (2006) y de acuerdo a la metodología del FMI, el ingreso estructural se expresa mediante la siguiente expresión:

$$
R_{S, t}=R_{C, t}\left(\frac{Y^{\prime}{ }_{t}}{Y_{t}}\right)^{\varepsilon}+R_{S, t}
$$

donde, $R_{C, t}$ expresa los ingresos fiscales en términos reales, $\varepsilon$ es la elasticidad de los ingresos tributarios con respecto a la producción, $Y_{t}^{\prime}$ representa el producto potencial y $R_{S, t}$ denota los ingresos independientes del ciclo económico.

De igual manera, siguiendo a Basso (2006), se parte del supuesto de la existencia de un componente estructural y un componente cíclico en los gastos del gobierno central. Por tanto es necesario estimar también la elasticidad del gasto corriente con respecto a la producción nacional, utilizando la ecuación (7):

$$
\operatorname{Ln}\left(E_{i, t}\right)=\alpha+\gamma_{i} \operatorname{Ln}\left(Y_{t}\right)
$$

4 Marcel et al. (2001) utilizó una elasticidad de los ingresos rezagada, considerando que algunos impuestos se recogen con un rezago en el periodo. Esta investigación no utiliza una elasticidad de los ingresos rezagada, debido a que se agregó en la ecuación original un rezago para el componente del PIB, expresado como: $\operatorname{Ln}\left(R_{i, t}\right)=\alpha+\varepsilon_{i} \operatorname{Ln}\left(Y_{t}\right)+\theta_{i} \operatorname{Ln}\left(Y_{t-1}\right)+\delta_{i, t}$, donde el coeficiente $\theta_{i}$ no resultó estadísticamente significativo, lo cual implica que no se pudo comprobar un rezago en la colecta de los ingresos tributarios. 
donde, $Y_{t}$ es igual al producto efectivo real, $E_{i, t}$ es igual al gasto corriente en términos reales, $\gamma_{i}$ es equivalente a la elasticidad del gasto corriente con respecto a la producción. Se realizó la estimación de la ecuación 7, detectando problemas de autocorrelación. Una posible fuente de autocorrelación es el efecto de la crisis sub-prime, por lo que se procedió incluir una variable dummy que recoja los efectos de la crisis. El resultado se aprecia en la siguiente ecuación:

$$
\operatorname{Ln}\left(E_{i, t}\right)=\alpha+\gamma_{i} \operatorname{Ln}\left(Y_{t}\right)+\theta_{i, t}
$$

donde, $Y_{t}$ es igual al producto efectivo real, $E_{i, t}$ es igual al gasto corriente en términos reales, $\gamma_{i}$ es equivalente a la elasticidad del gasto corriente con respecto a la producción y $\theta_{i, t}$ es la variable dummy que recoge los efectos de la crisis tomando el valor de 1 los años 2007 y 2008 y 0 el resto de los años. Al incorporar la variable dummy, se soluciona el problema de autocorrelación, por lo que se comprueba que dicho problema se origina de los efectos pre y post de la crisis sub-prime. El Anexo 2 refleja las estimaciones de la ecuación 7 y 8.

Siguiendo a Basso (2006), el gasto estructural se expresa mediante la siguiente expresión:

$$
E_{S, t}=E_{C, t}\left(\frac{Y_{t}^{\prime}}{Y_{t}}\right)^{\gamma}+E_{S, t}
$$

donde, $E_{C, t}$ expresa los gastos corrientes en términos reales, $\gamma$ es la elasticidad del gasto corriente con respecto a la producción, $Y_{t}^{\prime}$ representa el producto potencial y $E_{S, t}$ denota los gastos independientes del ciclo económico.

Ambas elasticidades se calcularon con datos anuales desde 1998 hasta 2014.

Consecuentemente, el balance estructural puede expresarse como:

$$
B_{S, t}=\left(R_{C, t}\left(\frac{Y_{t}^{\prime}}{Y_{t}}\right)^{\varepsilon}+R_{S, t}\right)-\left(E_{C, t}\left(\frac{Y_{t}^{\prime}}{Y_{t}}\right)^{\gamma}+E_{S, t}\right)
$$

Se utiliza la ecuación (9) para calcular el balance financiero, en donde $R_{C, t}$ representa los ingresos tributarios y $R_{S, t}$ el componente estructural, que es la suma de los ingresos no tributarios, transferencias corrientes e ingresos de capital. Por otro lado, $E_{C, t}$ expresa los gastos corrientes en términos reales y $E_{S, t}$ denota los gastos de capital.

Siguiendo a Basso (2001), al dividir ambos lados de la ecuación (10) por $Y^{\prime}$, resulta el balance estructural como porcentaje del PIB potencial.

$$
\frac{B_{S, t}}{Y^{\prime}{ }_{t}}=\frac{\left(R_{C, t}\left(\frac{Y_{t}^{\prime}}{Y_{t}}\right)^{\varepsilon}+R_{S, t}\right)}{Y^{\prime}{ }_{t}}-\frac{\left(E_{C, t}\left(\frac{Y_{t}^{\prime}{ }_{t}}{Y_{t}}\right)^{\gamma}+E_{S, t}\right)}{Y^{\prime}{ }_{t}}
$$

Para estimar el balance estructural primario como porcentaje del PIB potencial, en la ecuación (11), $R_{C, t}$ representa los ingresos tributarios, $R_{S, t}$ la suma de los ingresos no tributarios y las transferencias corrientes, $E_{C, t}$ son los gastos corrientes y se debe omitir el componente $E_{S, t}$ que serían los gastos de capital. 


\section{El Filtro Hodrick-Prescott}

En esta investigación se utilizó la metodología del Filtro Hodrick-Prescott (FHP), con el fin de estimar el producto potencial costarricense (Ver gráfico 2). El siguiente texto se extrajo de Basso (2006):

El FHP consiste en efectuar un suavizado de la serie en cuestión, que permite obtener una tendencia alrededor de la cual fluctúa el producto observado. Técnicamente, el método minimiza la varianza del producto $Y$ en torno a su valor de tendencia $Y^{\prime}$, sujeto a una restricción sobre $Y^{\prime}$. Es decir:

$$
\min \sum_{t=1}^{T}\left(Y_{t}-Y^{\prime}{ }_{t}\right)^{2}+\lambda \sum_{t=2}^{t-1}\left[\left(Y^{\prime}{ }_{t+1}-Y^{\prime}{ }_{t}\right)-\left(Y^{\prime}{ }_{t}-Y^{\prime}{ }_{t-1}\right)\right]^{2}
$$

donde $\lambda$ es un parámetro que determina el grado se suavización. Mientras mayor es $\lambda$, más suavizada es la serie. La elección del mismo es arbitraria y, para datos anuales, Hodrick y Prescott recomiendan un valor de $\lambda=100$. En este trabajo, se estimó el FHP con datos trimestrales y un parámetro $\lambda$ de 1600. Una vez estimados los datos ${ }^{5}$ en su forma trimestral, se sumaron para obtener la base anual.

El componente tendencial del PIB determina lo que en este artículo se denomina producto potencial, aunque puede decirse que representa el valor de la tendencia de largo plazo de la producción nacional. Entre las ventajas de este método, destaca su simplicidad y el hecho de que se requieren pocos datos para su cálculo, a diferencia de las estimaciones del producto potencial mediante la utilización de una función de producción. Su limitación más importante es que se trata de una técnica mecánica, insatisfactoria desde el punto de vista macroeconómico, puesto que no permite incorporar información referida a la estructura de la economía; ya que, por ejemplo, no permite modelar las posibles limitaciones y restricciones de factores que puedan existir.

GRÁFICO 2

COSTA RICA: PIB POTENCIAL Y REAL DE 1998 AL 2014

(EN MILLONES DE COLONES)

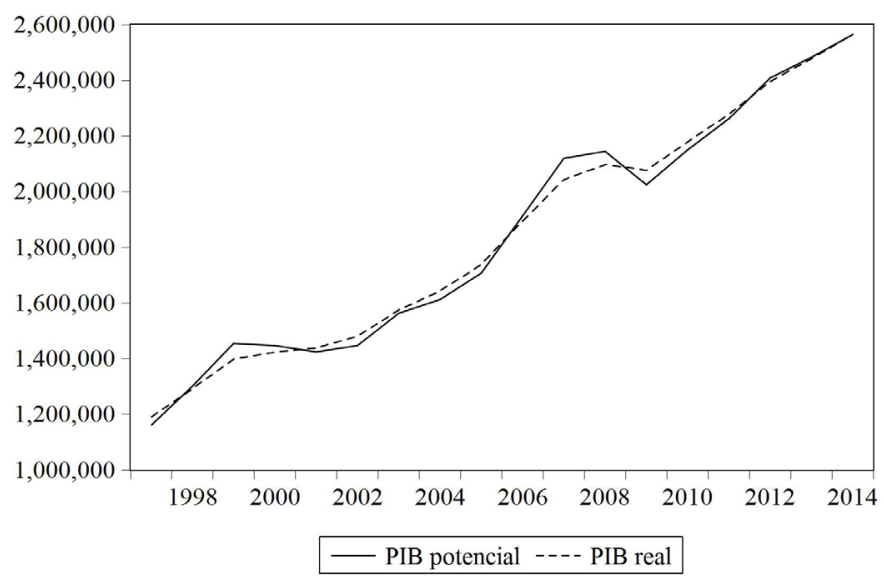

Fuente: Elaboración propia con base en información del Banco Central de Costa Rica.

5 El Producto Interno Bruto Real (PIB_real ), el logaritmo natural del PIB real, el PIB_real_sa (desestacionalizado), la tendencia Hodrick-Prescott (HP_trend) y el ciclo Hodrick-Prescott (HP_cycle), se trabajaron de forma trimestral, se sumaron para obtener la base anual, se calculó el Log(PIB_real_sa) y el Log(HP_trend) y finalmente se estimó la brecha del producto. 


\section{RESULTADOS}

Los resultados obtenidos en la presente investigación muestran un interesante análisis sobre el comportamiento del balance estructural con respecto al convencional. Este análisis ayuda a determinar momentos en los que el uso de indicadores convencionales evitó percibir la real situación fiscal. Este fenómeno, por ejemplo, pudo provocar que el gobierno en momentos de superávit estructural mayor al convencional no aprovechara este margen para generar una mayor recaudación para disminuir los niveles de deuda o ahorrar recursos para futuros préstamos en periodos desfavorables.

Así mismo, la estrecha relación que hay entre el producto nacional y la variación presente en los ingresos y gastos del gobierno central permite exponer una importante alerta. Los resultados demuestran la existencia de un significativo componente estructural; al demostrarse diferencias mínimas entre el balance estructural y convencional. Estos resultados son los que dan lugar a espacios para que el gobierno haya tomado acciones correctivas o de control. Acciones cuyos resultados podrían apreciarse solo en un análisis de balance estructural.

\section{GRÁFICO 3}

COSTA RICA: BALANCE PRIMARIO CONVENCIONAL Y ESTRUCTURAL EN RELACIÓN CON PIB REAL Y POTENCIAL, RESPECTIVAMENTE, DE 1998 AL 2014 (EN PORCENTAJES)

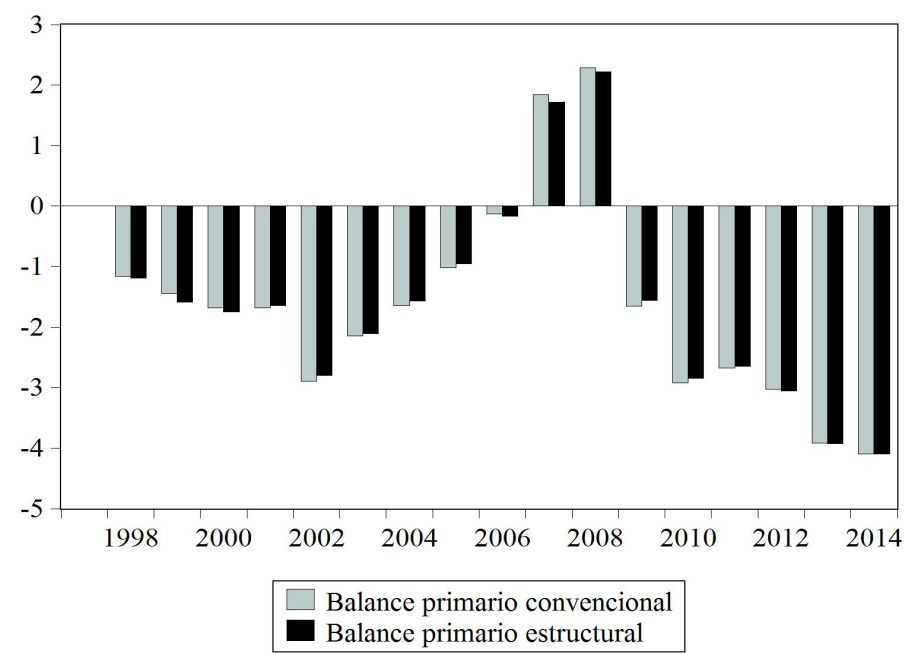

Fuente: Elaboración propia con base en información del Ministerio de Hacienda y el Banco Central de Costa Rica.

El gráfico 3 muestra que durante el periodo del 2001 al 2005 , el déficit fiscal estructural fue ligeramente menor que el resultado del balance convencional. Este comportamiento se debe a un aumento significativo en la brecha del producto negativa, en particular para los años 2002, 2007 y 2009. La brecha del producto positiva más grande ocurre en el año 2007 y las brechas negativas más grandes ocurren 2002 y 2009, como se aprecia en el gráfico 4. El gráfico 3 evidencia también que el balance convencional muestra el déficit primario más alto en el año 2014, debido al aumento de las variaciones anuales de la compra de bienes y servicios (8\%) y el pago de intereses (5\%). Sin embargo, los elementos con el mayor peso en el gasto corriente son el pago de sueldos y salarios y las transferencias corrientes, las cuales se ubican en 33,6\% y 39,9\%, respectivamente. 
Por otro lado, el mayor superávit convencional ubicado en 2008, es el resultado de un muy minúsculo aumento en el gasto corriente, especialmente en el gasto de intereses, cuya variación anual fue -29\%. Analizando el balance estructural, en el que la economía está en pleno uso de sus recursos, el mayor déficit se observa en 2014, con un 4,1\% del PIB. Por el contrario, el mayor superávit estructural para el periodo muestra fue en 2008, siendo este un $0,1 \%$ menor que el convencional; precisamente a causa de un PIB efectivo por encima de su potencial. Por último, se puede apreciar que, según el balance estructural, la economía de Costa Rica tuvo un superávit primario en 2006. Este excedente es el resultado de un mayor nivel de ingresos fiscales (1,3\%), en comparación con el balance convencional, $y$ a una brecha del producto positiva de $1,1 \%$.

GRÁFICO 4

COSTA RICA: BRECHA DEL PRODUCTO, DE 1998 AL 2014

(EN PORCENTAJES)

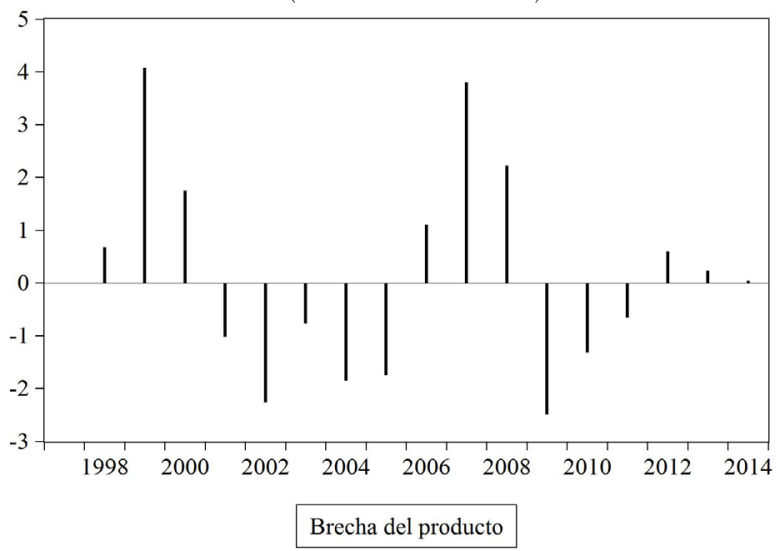

Fuente: Elaboración propia con base en información del Banco Central de Costa Rica.

\section{GRÁFICO 5}

COSTA RICA: VARIACIÓN INTERANUAL DEL PIB

REAL, INGRESOS ESTRUCTURALES Y GASTOS

ESTRUCTURALES, DE 1998 AL 2014 (EN PORCENTAJES)

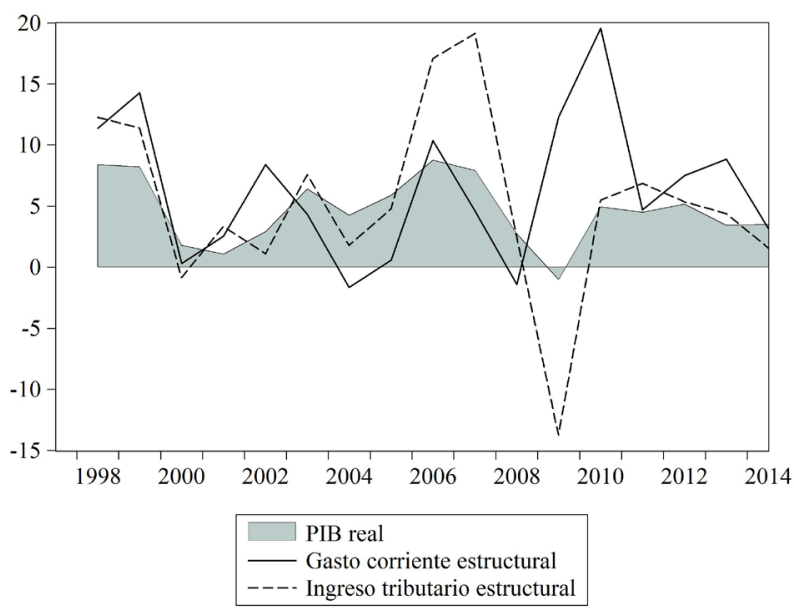

Fuente: Elaboración propia con base en información del Ministerio de Hacienda y el Banco Central de Costa Rica. 
Como se muestra en el gráfico 5, las tasas de variación siguen un patrón uniforme en todo el periodo de análisis, exceptuando solo el periodo post-crisis donde el gasto crece a tasas sobre el $20 \%$. Los ingresos presentan un crecimiento promedio para el periodo de un 5.3\%, sin embargo por el lado del gasto su crecimiento promedio es de un 7\%. Es importante destacar que la elasticidad de los ingresos tributarios con respecto a la producción nacional toma un valor de 1.08 y la elasticidad del gasto corriente con respecto a la producción nacional es de 1.31.

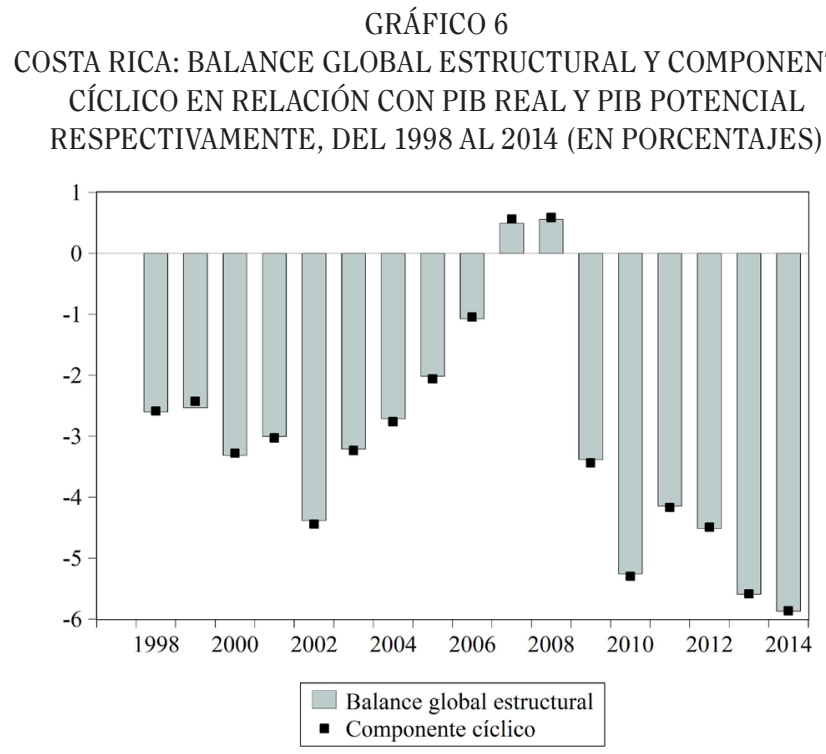

Fuente: Elaboración propia con base en información del Ministerio de Hacienda y el Banco Central de Costa Rica.

El gráfico 6 ilustra la tendencia del componente cíclico que viene a ser lo mismo que el balance global convencional, así como la tendencia del balance global estructural durante el periodomuestra y cómo el 2014 se ubica como el año con el déficit fiscal más alto, con un 5,9\% del PIB real y potencial. El déficit convencional más bajo ocurre en el año 2006, como resultado de una aceleración de la economía a partir de 2002. Por otro lado, se aprecia que durante los años 2007 y 2008 se contó también con un superávit fiscal en términos estructurales. Analizando el componente cíclico es fácil observar dónde estaría el balance global con una brecha de producción nula y que la existencia de un factor estructural permitió que se lograra un superávit en los años 2007 y 2008. Este factor estructural que generó el superávit se pudo aprovechar para enfrentar futuros compromisos o generar una mejor asignación de recursos. 


\section{CONCLUSIONES}

Este artículo presenta la primera aplicación de la metodología del Fondo Monetario Internacional en la estimación del balance estructural del Gobierno Central costarricense. A partir del presente estudio, se concluye lo siguiente:

1. Se evidencia la importancia de realizar análisis de política fiscal mediante la utilización de indicadores estructurales. Estos indicadores sobrepasan a los convencionales por una sencilla e importante razón; exponen la verdadera situación fiscal producto de las decisiones discrecionales del gobierno. Para el caso de Costa Rica, los resultados arrojan que el déficit fiscal es en esencia de naturaleza estructural. Esta consideración permite al gobierno tomar medidas correctivas necesarias en aras de mejorar la salud de las finanzas públicas, situación que no podría apreciar mediante análisis de indicadores convencionales.

2. Se comprobó que, en Costa Rica, durante todo el periodo muestra, el balance estructural se comporta de la misma forma que el producto efectivo cuando se aleja del potencial. Especialmente, para los años más recientes (2013 y 2014) la brecha del producto viene disminuyendo y las estimaciones arrojan los mayores déficits en el balance primario estructural para el periodo en muestra. Una de las razones de este fuerte deterioro fiscal es un incremento en el gasto público, acompañado de una caída en los ingresos tributarios.

3. Como se mencionó en la introducción, la metodología y los resultados de este trabajo difieren de los presentes en trabajos anteriores. Esto debido a la implementación de los coeficientes de sensibilidad del saldo público a la brecha del producto y de componente fijo estructural del saldo público, como en el caso de Armendáriz (2006).

Si se toma en cuenta que en este estudio se estimó una brecha del producto negativa, para el periodo de 1998 al 2003, que debe verse reflejada en una disminución del superávit estructural, o bien, un incremento en el déficit estructural y que Armendáriz (2006) y Ardanaz et al. (2015) muestran en sus resultados para ese mismo periodo un superávit estructural (de 1\% a 3,5\%), se deduce que estas investigaciones no ofrecen resultados compatibles con las características de la economía costarricense. Este trabajo investigativo sí muestra en sus resultados un déficit estructural acorde al comportamiento de la brecha del producto.

4. Con este estudio se evidencia la importancia de utilizar los datos apropiados para el cálculo del indicador para el caso de Costa Rica. En este trabajo se utilizaron datos fiscales a cierre de año publicados en la página oficial del Ministerio de Hacienda costarricense, los cuales son apropiados para el cálculo del balance estructural para el caso de Costa Rica. Sería incorrecto el empleo de los datos fiscales del presupuesto nacional de Costa Rica, dado que este sufre varias modificaciones por parte del Congreso a lo largo del año; lo que resulta en un presupuesto ejecutado diferente del que se aprueba. Adicionalmente, cuando se contrastaron los datos utilizados en esta investigación con los empleados por Ardanaz et al. (2015), se encontró que estos últimos ${ }^{6}$ no coinciden con las cifras de los presupuestos nacionales de Costa Rica ni con las cifras a cierre de año publicadas por el Ministerio de Hacienda costarricense. El uso de datos distintos a los oficiales agregado a una metodología que no concuerda con la problemática estructural evidenciada en este trabajo, descartan a Ardanaz et al. (2015) como una estimación apropiada para Costa Rica.

6 Los datos utilizados por Ardanaz et al. (2015) se puede encontrar en: http://publications.iadb.org/handle/11319/6979 y se pueden contrastar con los datos utilizados en la presente investigación; http:/www.hacienda.go.cr/contenido/141-ingreso-gasto-yfinanciamiento-del-gobierno-central-anual-desde-1987 
5. Es importante añadir que la interpretación del PIB potencial debe ser particularmente cauta, ya que por definición este no es una variable observable. Como se indica en Marcel et al. (2001, p. 26), es aconsejable considerar el PIB potencial como un valor aproximado, dada la incertidumbre inherente en su estimación. Por lo tanto, resulta indispensable actualizar el PIB potencial a medida que nueva información se encuentre disponible; por ejemplo, el cambio de año base del PIB real que en este momento realiza el BCCR. Del mismo modo, será necesario volver a estimar el balance estructural si el Ministerio de Hacienda de Costa Rica decide incluir entre sus partidas de gasto, el gasto por seguro de desempleo. Este gasto fluctúa con respecto al ciclo de negocios y, por tanto, se debe hacer una corrección a la metodología empleada en la presente investigación.

6. Por último, con este trabajo, el autor espera que este indicador de balance estructural sea una herramienta útil para el análisis fiscal de Costa Rica, ya que los análisis comunes se basan en proporciones de déficit/PIB o tasas de crecimiento del gasto corriente. Además, se pretende que este indicador pueda ser considerado en el desarrollo de una regla fiscal para Costa Rica, que proporcione claridad y transparencia en la formulación de las políticas fiscales que estimulen la estabilidad macroeconómica.

\section{ANEXOS}

ANEXO 1

ESTIMACIÓN DE LA ELASTICIDAD DE LOS INGRESOS TRIBUTARIOS CON RESPECTO A LA PRODUCCIÓN NACIONAL

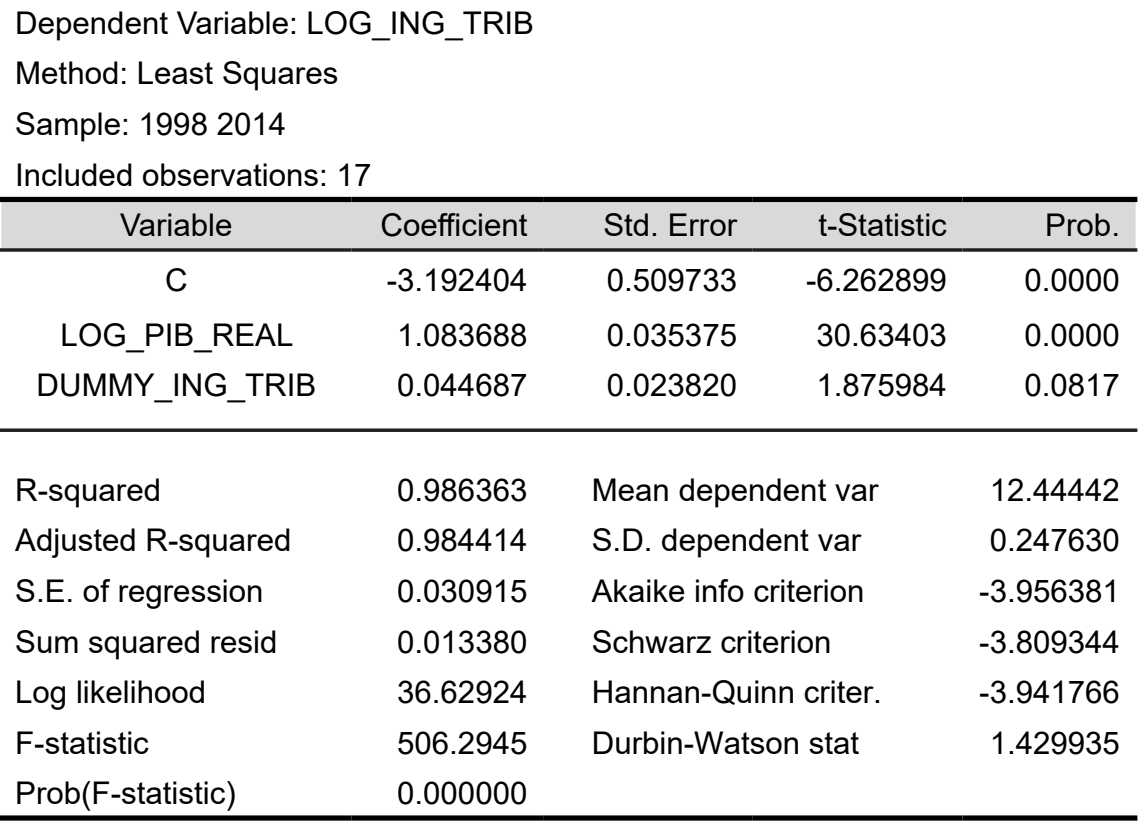


ANEXO 2

ESTIMACIÓN DE ECUACIÓN 7

Dependent Variable: LOG_GASTO_COR

Method: Least Squares

Sample: 19982014

Included observations: 17

\begin{tabular}{lrlrr}
\hline \multicolumn{1}{c}{ Variable } & Coefficient & Std. Error & t-Statistic & Prob. \\
\hline \multicolumn{1}{c}{ C } & -5.336398 & 1.600665 & -3.333864 & 0.0045 \\
\multicolumn{1}{c}{ LOG_PIB_REAL } & 1.241921 & 0.110957 & 11.19286 & 0.0000 \\
R-squared & 0.893071 & Mean dependent var & 12.57759 \\
Adjusted R-squared & 0.885943 & S.D. dependent var & 0.293883 \\
S.E. of regression & 0.099251 & Akaike info criterion & -1.672189 \\
Sum squared resid & 0.147763 & Schwarz criterion & -1.574164 \\
Log likelihood & 16.21361 & Hannan-Quinn criter. & -1.662445 \\
F-statistic & 125.2801 & Durbin-Watson stat & 0.514000 \\
Prob(F-statistic) & 0.000000 & & \\
\hline
\end{tabular}

Breusch-Godfrey Serial Correlation LM Test:

\begin{tabular}{|c|c|c|c|c|}
\hline F-statistic & 16.88067 & \multicolumn{2}{|c|}{ Prob. F(1,14) } & 0.0011 \\
\hline Obs*R-squared & 9.292914 & \multicolumn{2}{|c|}{ Prob. Chi-Square(1) } & 0.0023 \\
\hline \multicolumn{5}{|c|}{$\begin{array}{l}\text { Test Equation: } \\
\text { Dependent Variable: RESID } \\
\text { Method: Least Squares } \\
\text { Sample: } 19982014 \\
\text { Included observations: } 17 \\
\text { Presample missing value lagged residuals set to zero. }\end{array}$} \\
\hline Variable & Coefficient & Std. Error & t-Statistic & Prob. \\
\hline $\mathrm{C}$ & -0.334709 & 1.118555 & -0.299233 & 0.7692 \\
\hline LOG_PIB_REAL & 0.023484 & 0.077542 & 0.302848 & 0.7665 \\
\hline $\operatorname{RESID}(-1)$ & 0.763687 & 0.185875 & 4.108610 & 0.0011 \\
\hline R-squared & 0.546642 & \multicolumn{2}{|c|}{ Mean dependent var } & $-2.71 \mathrm{E}-15$ \\
\hline Adjusted R-squared & 0.481877 & \multicolumn{2}{|c|}{ S.D. dependent var } & 0.096100 \\
\hline S.E. of regression & 0.069173 & \multicolumn{2}{|c|}{ Akaike info criterion } & -2.345615 \\
\hline Sum squared resid & 0.066989 & \multicolumn{2}{|c|}{ Schwarz criterion } & -2.198577 \\
\hline Log likelihood & 22.93773 & \multicolumn{2}{|c|}{ Hannan-Quinn criter. } & -2.330999 \\
\hline F-statistic & 8.440336 & \multirow{2}{*}{\multicolumn{2}{|c|}{ Durbin-Watson stat }} & 1.184790 \\
\hline Prob(F-statistic) & 0.003936 & & & \\
\hline
\end{tabular}


ANEXO 3

ESTIMACIÓN DE ECUACIÓN 8

Dependent Variable: LOG_GASTO_COR

Method: Least Squares

Sample: 19982014

Included observations: 17

\begin{tabular}{lrlrr}
\hline \multicolumn{1}{c}{ Variable } & Coefficient & Std. Error & t-Statistic & Prob. \\
\hline \multicolumn{1}{c}{ C } & -6.334301 & 0.980905 & -6.457610 & 0.0000 \\
LOG PIB REAL & 1.313063 & 0.068069 & 19.29012 & 0.0000 \\
\hline \multicolumn{1}{c}{ DUMMYG } & -0.240381 & 0.045835 & -5.244476 & 0.0001 \\
R-squared & 0.963932 & Mean dependent var & 12.57759 \\
Adjusted R-squared & 0.958779 & S.D. dependent var & 0.293883 \\
S.E. of regression & 0.059667 & Akaike info criterion & -2.641287 \\
Sum squared resid & 0.049842 & Schwarz criterion & -2.494249 \\
Log likelihood & 25.45094 & Hannan-Quinn criter. & -2.626671 \\
F-statistic & 187.0753 & Durbin-Watson stat & 1.179817 \\
\hline Prob(F-statistic) & 0.000000 & & &
\end{tabular}

Prob(F-statistic)

0.000000

Breusch-Godfrey Serial Correlation LM Test:

\begin{tabular}{llll}
\hline F-statistic & 1.663856 & Prob. $F(2,12)$ & 0.2303 \\
\hline Obs ${ }^{*}$ R-squared & 3.690773 & Prob. Chi-Square(2) & 0.1580 \\
\hline
\end{tabular}

Test Equation:

Dependent Variable: RESID

Method: Least Squares

Date: 11/19/16 Time: 21:44

Sample: 19982014

Included observations: 17

Presample missing value lagged residuals set to zero.

\begin{tabular}{crlrr}
\hline Variable & Coefficient & Std. Error & t-Statistic & Prob. \\
\hline C & 0.011110 & 0.943073 & 0.011781 & 0.9908 \\
LOG_PIB_REAL & -0.000809 & 0.065416 & -0.012372 & 0.9903 \\
DUMMYG & 0.003403 & 0.065967 & 0.051586 & 0.9597 \\
RESID(-1) & 0.508021 & 0.285252 & 1.780953 & 0.1002 \\
RESID(-2) & -0.251736 & 0.408049 & -0.616926 & 0.5488 \\
\hline R-squared & 0.217104 & Mean dependent var & $-4.77 \mathrm{E}-15$ \\
Adjusted R-squared & -0.043861 & S.D. dependent var & 0.055813 \\
S.E. of regression & 0.057024 & Akaike info criterion & -2.650749 \\
Sum squared resid & 0.039021 & Schwarz criterion & -2.405686 \\
Log likelihood & 27.53136 & Hannan-Quinn criter. & -2.626389 \\
F-statistic & 0.831928 & Durbin-Watson stat & 1.953526 \\
Prob(F-statistic) & 0.530209 & & & \\
\hline
\end{tabular}




\section{AGRADECIMIENTOS}

El autor agradece a M.Sc. Henry Vargas y a Ph.D. Boris Segura por su contribución y orientación en este trabajo de investigación. De igual manera, a Ph.D. Rigoberto Torres por muchos años de invaluable apoyo.

Es prudente afirmar que los errores $u$ omisiones son estrictamente del autor y que las opiniones expresadas no representan necesariamente la posición de las instituciones a las que se encuentra afiliado.

\section{REFERENCIAS}

Ardanaz, M., Corbacho, A., Gonzales, A., \& Tolsa Caballero, N. (2015). Structural Fiscal Balances in Latin America and the Caribbean: New Dataset and Estimations (IDB Working Paper No. IDB-WP-579). Inter-American Development Bank. https://doi.org/10.18235/0000028

Armendáriz, E. (2006). La sostenibilidad de la deuda pública y la postura fiscal en el ciclo económico: el Istmo Centroamericano. México, D.F.: Naciones Unidas, CEPAL, Unidad de Desarrollo Económico.

Azofeifa. A., \& Rojas, D. (2000). Costa Rica: estimación déficit fiscal estructural cíclico. Banco Central de Costa Rica. (Documento de trabajo DM-DIE-PI-01-2000/R) Banco Central de Costa Rica, San José, Costa Rica. Recuperado de http://www.bccr.fi.cr/investigacioneseconomicas/sectorpublico/ Costa_Rica,_estimacion_deficit_fiscal_estructural_ciclico.pdf

Banco Central de Costa Rica (2014). Producción y Empleo. Recuperado de http://www.bccr.fi.cr/indicadores_economicos_Produccion_empleo.html

Basso, M. A. (2006). El Balance Estructural: Metodología y Estimación para Argentina. Anales de La Asociación Argentina de Economía Política.

Recuperado de http://www.aaep.org.ar/anales/works/works2006/BassoMartin.pdf

Bornhorst, F., Dobrescu, G., Fedelino, A., Gottschalk, J., \& Nakata, T. (2011). When and how to Adjust Beyond the Business Cycle?: A Guide to Structural Fiscal Balances. Technical Notes and Manuals, 11(02), 1. http://doi.org/10.5089/9781475510201.005

Burnside, C. (Ed.). (2005). Fiscal Sustainability in Theory and Practice: A Handbook. The World Bank. https://doi.org/10.1596/978-0-8213-5874-0

Federal Reserve Bank of Dallas. (s.f.). Deflating Nominal Values to Real Values - Dallas Fed. Recuperado de http://www.dallasfed.org/research/basics/nominal.cfm

International Monetary Fund. (octubre, 2015). World Economic Outlook Database. Recuperado de http://www.imf.org/external/pubs/ft/weo/2015/02/weodata/index.aspx

Hagemann, R. (1999). The Structural Budget Balance The IMF's Methodology (IMF Working Paper No. 99/95). International Monetary Fund.

Recuperado de https://www.imf.org/external/pubs/cat/longres.aspx?sk=3162.0

Marcel, M., Tokman, M., Valdes, R., \& Benavides, P. (setiembre, 2001). Balance estructural del Gobierno Central: metodología y estimaciones para Chile: 1987 - 2000. En A. Arenas \& M. Tokman (Eds.). Estudios de finanzas públicas.

Recuperado de http://www.dipres.gob.cl/594/articles-21639_doc_pdf.pdf

Marcel, M., Tokman, M., Valdés, R., \& Benavides, P. (diciembre, 2001). Balance estructural: la base de la nueva regla de política fiscal chilena. Economía Chilena, 4(3), 5-27. Recuperado de http://si2.bcentral.cl/public/pdf/revista-economia/2001/dic2001/recv4n3dic20015_27.pdf

Martner, R. (2000). Los estabilizadores fiscales automáticos. Revista de La CEPAL, 70, 31-52. Recuperado de http://repositorio.cepal.org/handle/11362/12198. 
Méndez, E., \& Durán, R. (1995). Orientación discrecional y cíclica de la política fiscal en Costa Rica. (Documento de trabajo DIE-PI-01-95/R) Banco Central de Costa Rica, San José, Costa Rica. Recuperado de. Recuperado de http://www.bccr.fi.cr/ndie/PI-01-1995R.PDF

Ministerio de Hacienda de Costa Rica. (2017). Ingreso, gasto y financiamiento del Gobierno Central (anual desde 1987). Recuperado de http://www.hacienda.go.cr/contenido/141-ingreso-gasto-yfinanciamiento-del-gobierno-central-anual-desde-1987

Okun, A. M. (1962). Potential GNP: its measurement and significance. En American statistical Association (Ed). Proceedings of the business and economic statistics section of the American Statistical Association. Volumen 7. (pp. 89-104).

Schinasi, G. J. \& Lutz, M. S. (1991). Fiscal Impulse. (Working Papers, 1991/91) International Monetary Fund. https://doi.org/10.5089/9781451851441.001 